\title{
Ceratoconjuntivite seca: Revisão sistemática
}

\author{
Keratoconjunctivitis seca: Systematic review \\ Queratoconjuntivitis seca: Revisión sistemática
}

Recebido: 18/06/2021 | Revisado: 23/06/2021 | Aceito: 08/07/2021 | Publicado: 18/07/2021

Thiago Vaz Lopes
ORCID: https://orcid.org/0000-0002-3192-1908
Universidade Federal do Acre, Brasil
E-mail: Thiagovlopes@ hotmail.com
João Gustavo da Silva Garcia de Souza
ORCID: https://orcid.org/0000-0002-5899-2490
Centro Universitário Aparício Carvalho, Brasil
E-mail: joaogarciadesouza27@ gmail.com
Jandira Santos da Silva
ORCID: https://orcid.org/0000-0001-5504-958X
Centro Universitário Aparício Carvalho, Brasil
E-mail: jandy.05@ @mail.com
Juliana Costa Rodrigues
ORCID: https://orcid.org/0000-0003-0712-7584
Hospital Veterinário Aparício Carvalho, Brasil
E-mail: julianamedvet_@ hotmail.com
Ellen Mireille Alvares
ORCID: https://orcid.org/0000-0001-8570-9892
Centro Universitário Aparício Carvalho, Brasil
E-mail: ellen.alvares.m@gmail.com
Iara Vaz Lopes
ORCID: https://orcid.org/0000-0003-0974-4615
Centro Universitário Aparício Carvallho, Brasil
E-mail: lopesiaravaz@ hotmail.com
Sandro de Vargas Schons

\section{Resumo}

A ceratoconjuntivite seca (CCS) ou síndrome do olho seco trata-se de uma afecção que se caracteriza de forma qualitativa e/ou quantitativa, sendo que geralmente ocorre em ambos os olhos (bilateral). Existem dois tipos de tratamento: o medicamentoso que consiste na utilização de lágrimas artificiais, imunossupressores, anti-inflamatório, antibióticos; e o tratamento cirúrgico em casos que não há resposta após a terapia medicamentosa. Este trabalho tem como objetivo buscar e avaliar os tratamentos da ceratoconjuntivite seca em cães que vem sendo utilizados nos últimos 15 anos, assim como a eficácia dos medicamentos e das técnicas cirúrgicas utilizadas, como também evidenciar a eficácia e importância da revisão sistemática. Foram selecionados 22 artigos, desses, 17 foram com base em tratamento medicamentoso, tendo como evidencia os imunossupressores (tacrolimus e ciclosporina, sirolimus), anti-inflamatório tópico (pimecrolimus), Etil-cianoacrilato e um inibidor de calcineurina, e cinco estudos selecionados foram utilizadas técnicas cirúrgicas, com diferentes eficácias de resultados. Baseado na literatura disponível e nos resultados da avaliação, os medicamentos utilizados apresentam eficácia em tratamentos por longo período; o transplante de glândulas salivares e o transplante de células mesenquimais têm mostrado resultados satisfatórios, a revisão sistemática tem a função de auxiliar o médico clínico na busca por novas alternativas de tratamentos.

Palavras-chave: Terapêutica; Técnicas cirúrgicas; Olho seco.

\begin{abstract}
The Keratoconjunctivitis sicca (KCS) or dry eye syndrome is a condition that is characterized in a qualitative and / or quantitative way, and it usually occurs in both eyes (bilateral). There are two types of treatment: medicated, which consists of using artificial tears, immunosuppressants, anti-inflammatory drugs, antibiotics; and surgical treatment in cases where there is no response after drug therapy. This work aims to seek and evaluate the treatments for dry keratoconjunctivitis in dogs that have been used in the last 15 years, as well as the efficacy of the medications and surgical techniques used, as well as to highlight the effectiveness and importance of the systematic review. Twenty-two articles were selected, of these, 17 were based on drug treatment, with immunosuppressants (tacrolimus and cyclosporine, sirolimus), topical anti-inflammatory (pimecrolimus), ethyl cyanoacrylate and a calcineurin inhibitor, and five selected studies were Surgical techniques are used, with different results efficacy. Based on the available literature
\end{abstract}


and the results of the evaluation, the drugs used are effective in long-term treatments; the transplantation of salivary glands and the transplantation of mesenchymal cells have shown satisfactory results, the systematic review has the function of assisting the clinical physician in the search for new treatment alternatives.

Keywords: Therapy; Surgical techniques; Dry eye.

\section{Resumen}

La queratoconjuntivitis seca (CCS) o síndrome del ojo seco es una condición que se caracteriza cualitativa y / o cuantitativamente, y suele presentarse en ambos ojos (bilateral). Hay dos tipos de tratamiento: el fármaco, que consiste en utilizar lágrimas artificiales, inmunosupresores, antiinflamatorios, antibióticos; y tratamiento quirúrgico en los casos en que no hay respuesta después de la terapia con medicamentos. Este trabajo tiene como objetivo buscar y evaluar los tratamientos de la queratoconjuntivitis seca en perros que se han utilizado en los últimos 15 años, así como la efectividad de los medicamentos y técnicas quirúrgicas empleadas, así como resaltar la efectividad e importancia de la revisión sistemática. Se seleccionaron veintidós artículos, de los cuales 17 se basaron en tratamiento farmacológico, teniendo como evidencia los inmunosupresores (tacrolimus y ciclosporina, sirolimus), antiinflamatorio tópico (pimecrolimus), cianoacrilato de etilo e inhibidor de la calcineurina, y se seleccionaron cinco estudios. Se utilizaron técnicas quirúrgicas, con diferente eficacia de resultados. Con base en la literatura disponible y en los resultados de la evaluación, los fármacos utilizados son eficaces en los tratamientos durante un período prolongado; El trasplante de glándulas salivales y el trasplante de células mesenquimales han mostrado resultados satisfactorios, la revisión sistemática tiene la función de ayudar al clínico en la búsqueda de nuevas alternativas de tratamiento.

Palabras clave: Terapéutica; Técnicas quirúrgicas; Ojo seco.

\section{Introdução}

A ceratoconjuntivite seca (CCS) ou olho seco é uma oftalmopatia grave e progressiva que pode se tornar crônica com o decorrer do tempo e comprometer a visão. Associada frequentemente com uma destruição imunomediada de glândulas lacrimais semelhante à síndrome de Sjögren (doença autoimune) em humanos (Ofri et al., 2007).

Considerada uma enfermidade comum em pequenos animais (principalmente em cães) caracterizada pela diminuição da porção aquosa da lágrima, que resulta no ressecamento e inflamação da córnea e da conjuntiva, na qual o cão tem um desconforto ocular e uma diminuição da atividade visual e dependendo da gravidade pode ocasionar cegueira (Pigatto et al., 2007).

Existem duas etiologias: a primária, que ocorrem devido a fatores genéticos (má formação da glândula), idade (deficiência na glândula na produção do filme lacrimal pré-corneal), causas também secundárias pelo uso prolongado de algumas drogas como as sulfonamidas, atropina, agentes anestésicos, doenças sistêmicas (cinomose), doenças metabólicas (diabetes mellitus, hipotireoidismo, hiperadrenocorticismo), traumas, doenças neurogênicas, tumores e a remoção da glândula da terceira pálpebra, entrópio e ectrópio (Gellat, 2003; Slatter, 2005).

A incidência de CCS em cães é de aproximadamente 1\% e acomete com maior frequência os cães das seguintes raças: Shihtzu, Lhasa Apso, Pequinês, Buldogue Inglês, Yorkshire Terrier, Pug, Cocker Spaniel Americano, West Highland White Terrier e Schnauzer miniatura (Pigatto et al., 2007).

O teste lacrimal de Schirmer é utilizado comumente para o diagnóstico da CCS em cães, mas existem outras técnicas que mensuram a produção lacrimal, porém não são muito utilizados na rotina da oftalmologia veterinária (Angélico et al., 2011).

O tratamento mais indicado é o medicamentoso, no qual incluem os imunossupressores (ciclosporina, tacrolimus, pimecrolimus), lágrimas artificiais, antibióticos, anti-inflamatórios, óleo de linhaça (Berdoulay, 2005; Pigatto et al., 2007).

Objetivou-se com essa revisão sistemática reunir e analisar criteriosamente os tratamentos clínicos e cirúrgicos modernos e recentemente publicados, utilizados para o tratamento da ceratoconjuntivite seca em cães, através dos bancos de informações científicas, disponíveis em forma de artigo como também demonstrar a aplicabilidade dessa metodologia na medicina veterinária. 


\section{Metodologia}

\subsection{Estratégia de busca}

O caminho metodológico procedeu-se com a pesquisa documental de artigos publicados nos últimos quinze anos, nas bases de dados: Scielo, Pubmed, Lilacs e Google Acadêmico, formando um trabalho de natureza quantitativa. Com os seguintes termos: "ceratoconjuntivite seca", em uma combinação de pesquisa com os seguintes termos identificadores da espécie: "cães", "canina" e com o termo "tratamento".

\subsection{Critérios de inclusão}

Estudos clínicos que apresentaram os tratamentos da ceratoconjuntivite seca em cães, bem como o efeito de medicamentos; investigação comparando a eficácia entre medicamentos, técnicas cirúrgicas utilizadas atualmente e uso de transplantes de glândulas salivares. Foram considerados os artigos em português e inglês publicados nos últimos 15 anos.

\subsection{Critérios de exclusão}

Estudos experimentais com modelo murino, os que aliavam resultados histológicos e artigos publicados há mais de 15 anos.

\subsection{Extração dos dados}

Foram selecionados artigos que preenchem os critérios de inclusão, constando dados relevantes e com características referentes ao conteúdo sobre os tipos de tratamentos. Os seguintes dados foram extraídos: nomes dos autores, ano da publicação, os tipos de tratamentos, quais medicamentos foram mais utilizados, efeitos positivos, quais cirurgias foram realizadas, tipos de transplantes e duração do tratamento. Sendo um estudo avaliativo da qualidade das publicações como também de sua quantidade.

\section{Resultados}

Com base na metodologia empregada para a busca de artigos foram encontrados 43 artigos, sendo que desses 22 foram selecionados para análise e 21 não se encaixam nos critérios de inclusão. Dos selecionados, 17 utilizaram tratamentos terapêuticos e cinco utilizaram tratamentos cirúrgicos.

\subsection{Tratamento clínico}

Entre os 17 estudos que testaram medicamentos, notamos que desses, 12 autores utilizaram imunossupressores (ciclosporina, tacrolimus, sirolimus e o pimecrolimus).

Pimecrolimus foi utilizado em três estudos de Rorig, 2009; Nell, et al, 2005 e Ofri et al, 2007. Rorig (2009) fez administração dessa medicação em forma tópica $0,5 \%$, BID, durante 23 semanas, e a partir da $2^{\mathrm{a}}$ semana de tratamento foi observada diferença estatística nos valores de TLS. Já Nell e colaboradores (2005) utilizaram a $1 \%$ à base de óleo de milho (1 gota três vezes/dia por 4 a 32 semanas) na $1^{\text {a }}$ semana já houve resultados positivos, enquanto que OFRI et al. (2007) comparam o pimecrolimus $1 \%$ à base de milho (24 cães) com a ciclosporina $0,2 \%$ (20 cães) e após 8 semanas de tratamento o pimecrolimus mostrou-se superior.

Os autores Berdoulay,et al., 2005; Hendrix, et al., 2011; Radziejewski e Balicki, 2016; Destefanis et al., 2016 avaliaram o tacrolimus, sendo que o Hendrix, et al. (2011) fizeram uma comparação com a ciclosporina $2 \%$, ambos foram dissolvidos em óleo de oliva, onde resultou que o tacrolimus $(0,03 \%)$ é superior à CsA; enquanto Berdoulay et al. (2005) utilizaram o tacrolimus 
a $0,02 \%$ (tópico), no qual foi dividido em estudo de eficácia inicial e estudo controlado, ambos demonstraram aumento significativo nos valores de TLS, resultando positivamente.

Radziejewski e Balicki (2016) também avaliaram o tracolimus (0,02\%) BID em comparação a ciclosporina (0,75\%) 3 vezes ao dia, onde, de maneira geral, ambos apresentaram melhora significativa em relação à redução dos sinais clínicos, redução do exsudato purulento do saco conjuntival, aumento dos valores de STT, diminuição da neovascularização e redução no grau de pigmentação.

Destefanis et al. (2016) empregaram o tracolimus (0,03\%) BID em associação a uma dieta nutracêutica durante 60 dias. Este estudo evidenciou melhora significativa dos animais tratados com tracolimus associado à essa dieta em relação aos animais tratados apenas com tracolimus.

A ciclosporina foi administrada isoladamente no estudo desenvolvido por Souza e Renault (2014), onde o cão foi tratado por um período de 30 dias, mas não apresentou melhora. Uma associação, com a ciclosporina foi visto na pesquisa de Almeida, et al., 2004; Barsotti e Vezzosi, 2016 e Lee et al., 2018.

No estudo de Almeida, et al. (2004), a ciclosporina 1\% (BID) foi associada ao Ácido poliacrílico (TID), contudo, neste estudo os resultados foram insatisfatórios. A pesquisa de Barsotti e Vezzosi (2016) realizaram testes relacionando o uso da ciclosporina 0,2\% (BID) com o Hialorunato de Sódio 0,4\% (QID), obtendo resultados excelentes.

Neste estudo, o paciente após dois meses foi diagnosticado com estenose pulmonar e precisou ser tratado com Atenolol, e o que os pesquisadores observaram foi a piora exacerbada do quadro de ceratoconjuntivite seca, que posteriormente foi completamente revertida após a interrupção da administração do Atenolol.

Lee et al. (2018) comparam a eficácia da ciclosporina 1\% diluída em óleo de milho (BID) em relação a HL036, nas doses de $0,2 \mathrm{mg} / \mathrm{ml}, 1 \mathrm{mg} / \mathrm{ml} \mathrm{e} 5 \mathrm{mg} / \mathrm{ml}$ (BID). Os resultados obtidos revelaram eficácia nos tratamentos com as três concentrações, de maneira que houve redução dos sinais clínicos e aumento da produção de lágrima em longo prazo.

Gilger et al. (2013) conduziram o estudo com inibidor de calcineurina tópica - SCY-641 (derivado da ciclosporina) que mostrou ser eficaz para o tratamento de ceratoconjuntivite em cães.

O etil-cianoacrilato foi testado por Rosa (2011) através da obstrução do ducto nasolacrimal em 11 cães, houve inflamação local na qual foi controlada com diclofenaco sódico. Em relação ao resultado foi observado aumento de retenção de lágrima na superfície independente da produção, no qual mostrou um bom resultado.

Williams et al. (2012) administraram colírio tópico a base de Acido Hialurônico em 27 cães e realizaram testes avaliadores antes do início do tratamento e na $2^{\mathrm{a}}$ e 4 semanas após o início dele, reduzindo a hiperemia conjuntival e da irritação ocular.

Na pesquisa desenvolvida por Chen e Powell (2015) o tratamento foi realizado através da lavagem ocular com solução salina comercial ou com solução de naltrexona a $0,3 \%$. Nas semanas 1,2 e 4 os animais foram avaliados. Na semana 4 o tratamento foi descontinuado e na semana 5 foram reavaliados. Contudo, o tratamento não encontrou diferenças estatisticamente entre os tratamentos utilizados na avaliação.

Spatola et al. (2017) compararam os efeitos do Sirolimus 0,02\% em cães saudáveis e cães com CCS (Ceratoconjuntivite Seca). Nos cães saudáveis a administração do sirolimus era feita BID por quatro semanas, enquanto que no grupo de animais portadores da CCS, a aplicação era feita ou com sirolimus 0,02\% ou tracolimus 0,02\% BID. Desta forma, os autores observaram aumento da produção de lágrima com sirolimus no grupo dos cães saudáveis, bem como aumento da produção de lágrima dos animais do grupo com CCS, tanto nos tratados com sirolimus quanto nos tratados com tracolimus, não havendo diferencias estatísticas entre os grupos.

No trabalho de Bonini, et al. (2005) foi administrada pomada tópica a base de Fator de Crescimento Nervoso (NGF) BID por 30 dias em três Bulldogs ingleses, após a retirada intencional da glândula de $3^{\mathrm{a}}$ pálpebra bilateral, sendo um olho 
(experimental) tratado com pomada NGF e o olho (controle) foi apenas com veículo à base de vaselina. Esse tratamento evidenciou melhora do componente mucoso da lágrima do olho experimental quando comparado ao controle.

\subsection{Tratamento cirúrgico}

Dos artigos analisados, Angélico et al., (2011), Rocha, (2012), Castanho et al., (2013), e Barachetti et al., (2015) utilizaram tratamentos cirúrgicos na busca pela resolução da CCS, os 3 primeiros utilizaram a técnica de transplante de glândula salivares.

Foi realizado o transplante de glândulas salivares menores em 16 olhos, sendo que desses 15 apresentaram melhora, mostrando resultado satisfatório no experimento de Angélico et al. (2011). Foram realizados transplantes de glândulas salivares labiais em 17 cães, de forma eficiente no estudo desenvolvido por Castanho et al. (2013).

Já na pesquisa feita por Rocha (2012) foi realizado um transplante autólogo das glândulas salivares labiais, oclusão cirúrgica dos pontos lacrimais ou associação das duas técnicas em 16 cães (27 olhos), de acordo com a melhora dos sinais clínicos em relação aos grupos, obtiveram-se resultados moderado a bom.

O uso do implante de ciclosporina episclerais (ESMC) foi avaliado em 15 cães ( 27 olhos) pelo estudo de Barachetti et al. (2015), os implantes foram bem tolerados por todos os cães, porém, de acordo com os autores, ainda há a necessidade de se conduzirem novos estudos a fim de determinar a eficácia e dose.

Bittencourt et al. (2016) demonstraram em seu trabalho o transplante de células mesenquimais alogênicas em cães com CCS. Essas células foram coletadas de amostras de gordura visceral e transplantadas com auxílio de seringa de $1 \mathrm{ml}$ para região anatômica da glândula dorsal e da glândula de $3^{\mathrm{a}}$ pálpebra. Nos olhos receptores do transplante também foram aplicadas lágrimas artificiais três vezes ao dia por 30 dias. Os resultados obtidos foram bastantes significativos, evidenciando melhora mesmo após 12 meses da realização do tratamento.

\section{Discussão}

Os resultados qualitativos desta revisão mostraram a eficácia dos tratamentos utilizados para a CCS em cães, quanto ao tratamento clínico Berdoulay et al, 2005 defendem os imunossupressores (ciclosporina e tacrolimus) como os mais preconizados, porém de custo elevado. E que o uso de formulações tópicas (álcool povinilico 1,4\% + acetilcisteína 10\% + pilocarpina 1\%) entrou em desuso, apesar de ser uma opção boa e mais acessível para proprietários carentes que tenham cães portadores da oftalmopatia.

Um estudo apresentado por Nell et al. (2005), demonstrou que o pimecrolimus a 1\% preparado com óleo de milho, causou aumento significativo dos valores de TLS, outro estudo evidenciou a eficácia do pimecrolimus (1\%) no controle das inflamações coreana, conjuntival e na estimulação da produção lacrimal.

De acordo com os resultados dos estudos, o tacrolimus e pimecrolimus possuem efeito superior em relação à ciclosporina. Apenas um estudo (Spatola et al., 2017) utilizou o sirolimus 0,02\% em seus experimentos, e obteve resultados positivos similares aos obtidos com tracolimus, porém mais estudos serão necessários para uma melhor avaliação.

Quanto aos tratamentos cirúrgicos utilizados os autores Angélico (2011) e Castanho et al. (2013) ressaltam a eficácia do transplante de glândula salivares, porém esse tipo de tratamento só é indicado em casos não responsivos aos tratamentos clínicos. Um estudo realizado por Bittencourt et al. (2016) sobre transplante de células mesenquimais alogênicas demonstrou resultados positivos bastantes significativos, desta forma, podem ser considerados boa técnica para tratamento desta oftalmopatia.

Em relação à aplicação clínica e cirúrgica dos resultados apresentados nesta revisão, notamos que apesar da eficácia de alguns medicamentos e técnicas cirúrgicas, ainda é necessário mais estudos como, por exemplo, a utilização do óleo da semente 
de linhaça e Omega 3 e 6, como mencionado por Destefanis et al. (2013) e comparações significativas entres os tratamentos, em caso de insucesso do tratamento clinico medicamentoso, faz-se necessário o tratamento cirúrgico (transplante de glândulas salivares ou transplante de células mesenquimais alogênicas).

Sendo assim quantitativamente, foram selecionados 43 artigos durante as pesquisas, e após a aplicação dos critérios de inclusão e exclusão, os números a serem trabalhados mostraram que 22 (51\%) deles foram satisfatórios sendo então 21 (49\%) excluídos. Quando comparado com a revisão sistemática apresentada por Camargo (2013), em que foram revisados 1628 artigos, com a utilização de apenas cinco $(0,30 \%)$, após a aplicação dos critérios de inclusão e exclusão, apresentando assim um número inferior a essa pesquisa, quando comparado ao número de utilizados, embora no geral houvesse um volume superior.

Nogueira (2015) pesquisou 173 artigos e 45\% (78 artigos) foram selecionados para o estudo de revisão sistemática, com isso notau-sse que após uma ampla busca, a quantidade de artigos utilizados por ele corrobora com os utilizados por este artigo.

Outras revisões apresentadas por Decurcio (2007), de 303 artigos pesquisados e 71 selecionados, como também a de Navarro (2012), com 51 artigos encontrados e 12 selecionados, tiveram os mesmos resultados em termos de porcentagem média de aproveitamento, estando próximo aos $23 \%$ os que foram porem eles trabalhados.

\section{Conclusão}

Após os resultados apresentados, podemos concluir que o pimecrolimus e o tacrolimus estão sendo cada vez mais utilizados, pois apresentaram resultados satisfatórios em relação à ciclosporina e outros medicamentos (lágrima artificial, pilocarpina) utilizados em cães com CCS.

Os estudos recentes, com o uso do Inibidor de calcineurina, o etil-cianoacrilato e o sirolimus apresentam um acréscimo para o leque de tratamentos, sendo bem tolerados pelos cães, assim como a associação destes fármacos com dieta nutracêutica.

Sendo assim ainda para os casos de insucesso ao tratamento clínico medicamentoso, a opção do transplante de glândulas salivares ou o transplante de células mesenquimais alogênicas, por enquanto a utilização de implantes com ciclosporina episcleral, ainda ficou ao encargo de mais estudos assegurar a sua eficácia.

Esta revisão sistemática traz a demonstração dos medicamentos mais utilizados e o grau de eficácia deles, segundo os artigos e experimentos pesquisados assim como as técnicas cirúrgicas que mostraram resultados positivos sendo bem tolerados para resolução ou estabilização das lesões, sempre visando o bem estar do paciente.

Com isso, a revisão sistemática da ceratoconjuntivite seca se mostra importante, pois absorve todos os trabalhos anteriores sobre o tema afim de mostrar compatibilidades entre eles, ao mesmo tempo em que deixa aberturas para que no futuro outros trabalhos sobre o tema sejam relevantes, afim de melhorar cada vez mais o sucesso dos tratamentos utilizados para a CCS.

\section{Referências}

Almeida, D. E., et al. (2004). Iatrogenic keratoconjunctivitis sicca in a dog. Ciência Rural, 34(3): 921-924.

Angélico, G. T., et al. (2011). Transplante de glândulas salivares menores no tratamento da ceratoconjuntivite seca em cães. Arquivo Brasileiro de Medicina Veterinária e Zootecnia, 63(5): 1087-1092.

Barachetti, L., et al. (2015). Use of episcleral cyclosporine implants in dogs with keratoconjunctivitis sicca: pilot study. Veterinary Ophthalmology, 18(3):234241. 10.1111/vop. 12173

Barabino, S., Rolando, M. \& Dana, R. (2004). Tear film and ocular surface tests in animal models of dry eye: uses and limitations. Experimental Eye Research, 79(5): 613-21.

Barabino et al. (2003). Systemic Linoleic and $\gamma$-Linolenic Acid Therapy in Dry Eye Syndrome With an Inflammatory Component. Cornea, $22(2)$ : 97-101.

Barsotti, G. \& Vezzosi, T. (2016). Keratoconjunctivitis sicca exacerbation in a dog treated with systemic atenolol. Journal of Small Animal Practice, 57(7): 379381. 10.1111/jsap.12477 
Berdoulay, A., English, R. V. \& Nadelstein, B. (2005). Effect of topical 0,02\% tacrolimus aqueous suspension on tear production in dogs with keratoconjunctivitis sicca. Veterinary Ophtalmology, 8(4): 225-232.

Bittencourt, M. K. W., et al. (2016). Allogeneic Mesenchymal Stem Cell Transplantation in Dogs With Keratoconjunctivitis Sicca. Cell Medicine, 8(3): 63-77. http://dx.doi.org/10.3727/215517916X693366

Coassin, M., et al. (2005). Efficacy of topical nerve growth factor treatment in dogs affected by dry eye. Graefe's Arch. Clin. Exp. Ophthalmol., 243(2): 151155. 10.1007/s00417-004-0955-2

Castanho, L. S., et al. (2013). Transplante de Glândulas Salivares Labiais no Tratamento de Olho Seco em Cães pela Autoenxertia. Revista Brasileira Oftalmologia. 72(6): 373-378.

Castro, A. P. B. M. (2006). Inibidores de Calcineurina no tratamento das dermatoses alérgicas. Jornal de Pediatria, 82(5).

Chen, T. \& Powell, C. C. (2015). Effect of once daily topical $0.3 \%$ naltrexone on tear parameters and corneal sensitivity in dogs with uncontrolled keratoconjunctivitis sicca: a double-masked randomized placebo-controlled clinical trial. Veterinary Ophthalmology, 18(6): 497-450. DOI:10.1111/vop.1225

Cunha, O., et al. (2002). Ceratoconjuntivite Seca. Revista Nosso Clínico, 5(28): 6-10.

Decurcio, D. A. (2007). Estudos longitudinais da influência do veículo na eficácia da pasta de hidróxido de cálcio em infecções endodônticas - revisão sistemática. Dissertação (Mestrado em Ciências da Saúde) - Universidade Federal de Uberlândia.

Defante junior, A. (2006). Ceratoconjuntivite seca em cães. Monografia (Pós-Graduação) - Universidade Castelo Branco.

Destefanis, S., et al. (2016). Clinical evaluation of a nutraceutical diet as an adjuvant to pharmacological treatment in dogs affected by Keratoconjunctivitis sicca. BMC Veterinary Research, 12(214): 1-12. 10.1186/s12917-016-0841-2

Garcia, S. C., et al. (2004). Ciclosporina e Tacrolimus: Uma Revisão. Bras. Patol. Med. Lab., 40(6): 393-401.

Gilger, B. C., et al. (2013). A topical aqueous calcineurin inhibitor for the treatment of naturally occurring keratoconjunctivitis sicca in dogs. Veterinary Ophthalmology, 16(3): 192-197. 10.1111/j.1463-5224.2012.01056.x

Giuliano, E. A. \& Moore, C. P. (2007). Diseases and surgery of the lacrimal secretory sistem. In: GELATT, K. N. Veterinary Ophthalmology. (4a ed.), Blackwell publishing, 2(13): 633-661.

He, J. \& Bazan, H. E. P. (2010). Omega-3 Fatty acids in Dry Eye and Corneal Nerve Regeneration after Refractive Surgery. Prostaglandins Leukot Essent Fatty Acids, 82(4-6): 319-325.

Hendrix, D. V. H., et al. (2011). An Investigation Comparing the Efficacy of Topical Ocular Application of Tacrolimus and Cyclosporine in Dogs. Veterinary Medicine International, 10.4061/2011/487592.

INPA. (2006) Instituto Nacional de Proteção Animal.

Kierszenbaum, A. L. \& Tres, L. L. (2012). Histologia e Biologia Celular. (3a ed.), Elsevier.

Kleiner, J. A. (2003). Tratamento Cirúrgico da Epífora Crônica em Animais de Companhia. Dissertação (Mestrado em Ciências Veterinárias) - Universidade Federal do Paraná.

Ribeiro, A. P., et al. (2008) Qualitative and quantitative tear film anormalities in dogs. Ciência Rural, 38(2). 10.1590/S0103-84782008000200048

Lee, H-B., et al. (2018). Efficacy of HL036 Versus Cyclosporine A in the Treatment of Naturally Occurring Canine Keratoconjunctivitis Sicca. Current Eye Research, 43(7): 889-895.

Navarro, F. K. S. P., et al. (2012). Relação entre IGF-I e cortisol no crescimento de peixes teleósteos de produção: revisão sistemática. Revista científica eletrônica de medicina veterinária, 10(19).

Nell, B., et al. (2005). The effect of topical pimecrolimus on keratoconjunctivitis sicca and chronic superficial keratitis in dogs: results from an exploratory study. Veterinary Ophthalmology, 8(1): 39-46.

Nogueira, G. M. (2015) Terapia assistida por animais: revisão sistemática da literatura e análise exploratória da prática psicomotora. Dissertação (Mestrado em Reabilitação Psicomotora) - Universidade de Lisboa, Faculdade de Motricidade Humana, Lisboa.

Ofri, R., et al. (2007). Clinical evaluation of pimecrolimus eye drops for treatment of canine keratoconjunctivitis sicca: a comparasion with cyclosporine A. The Veterinary Journal, 179(1): 70-77.

Perlmann, E. \& Almeida, A. C. V. R. (2011). Anatomia e Fisiologia Ocular - Revisão de Literatura. Medvep - Revista Cientifica de Medicina Veterinária Pequenos Animais e Animais de Estimação, 9(30): 410-19.

Pigatto, J. A., et al. (2007). Ceratoconjuntivite Seca em Cães e Gatos. Acta Sciential, 35(2): 250-251.

Pimentel, T. S. C. (2013). Revisão sistemática: tratamento da osteoatrose com uso de anti-inflamatórios não esteroidais em cães. Dissertação (Mestrado em Ciência) - Faculdade de Medicina da Universidade de São Paulo, São Paulo.

PINHEIRO JUNIOR, M. N., et al. (2007). Uso oral do óleo de linhaça (Linumus it atissimum) no tratamento do olho seco de pacientes portadores da síndrome de Sjögren. Arq. Bras. Oftalmol. 70(4): 649-655.

Pinney, C. C. (2004). The Complete Home Veterinary Guide. (3a ed.), 305-306. 
Research, Society and Development, v. 10, n. 8, e56510817354, 2021

(CC BY 4.0) | ISSN 2525-3409 | DOI: http://dx.doi.org/10.33448/rsd-v10i8.17354

Radziejewski, K. \& Balicki, I. (2016). Comparative clinical evaluation of tacrolimus and cyclosporine eye drops for the treatment of canine keratoconjunctivitis sicca. Acta Veterinaria Hungarica. 64(3): 313-329. 10.1556/004.2016.030

Rocha, G. L. S. (2012). Transplante Autólogo das Glândulas Salivares Labiais, Oclusão Cirúrgica dos Pontos Lacrimais ou Associação das Duas Técnicas no Tratamento de Ceraconjuntivite Seca em Cães. Tese (Doutorado em Cirurgia e Oftalmologia) - Faculdade de Medicina, Universidade de Minas Gerais.

Roncone, M., Bartlett, H. \& Eperjesi, F. (2010). Essential fatty acids for dry eye: A review. Contact Lens \& Anterior Eye, 33(2): 49-54.

Rorig, M. C. L. (2009). Uso de Pimecrolimus 0,5\% no Tratamento da Ceratoconjuntivite Seca em Cães. Dissertação (Mestrado em Ciências Veterinárias) Universidade Federal do Paraná.

Rosa, A. S., Pereira, J. T. \& Botteon, R. C. C. M. (2012). Obstrução do duto nasolacrimal com etil-cianoacrilato como adjuvante no tratamento da ceratoconjuntivite seca em cães. Rev. Bras. Med. Vet., 34(4):353-360.

Sgrignoli, M. R. et al. (2013). Comparison of Topical 0.03\% Tacrolimus in Almond and Linseed Oil to Treat Experimentally In duced Keratoconjunctivitis Sicca in Rabbits. International Journal Ophthalmic Pathology, 2:3.

Souza, G. E. \& Renault, P. (2014). Ceratoconjuntivite Seca em Cão da Raça Pinscher. Relato de Caso. Faculdade Promove de Brasília, 10 p.

Spatola, R., et al. (2018). The effects of topical aqueous sirolimus on tear production in normal dogs and dogs with refractory dry eye. Veterinary Ophthalmology, 21(3): 255-263. 10.1111/vop.12503

Stades, F. C., et al. (1999). Aparelho lacrimal. Fundamentos de Oftalmologia Veterinária. p. 54-8.

Taffarel, M. O., Cavalcante, C. Z. \& Cunha, O. (2002). Ceratoconjuntivite seca. Revista Nosso Clínico. 5(28): 6-12.

Tang-Liu, D. D. S. \& Acheampong, A. (2005). Ocular Pharmacokinetics and Safety of Ciclosporin, a Novel Topical Treatment for Dry Eye. Clin Pharmacokinety, 44(3): 247-261. 10.2165 / 00003088-200544030-00003

Tolosa, E. M. C \& Rodrigues, C. J. (2003). Manual de Técnica para Histologia Normal e Patológica. (2a ed.), Manole, 34 p.

Verna, C., et al. (2012). Síndrome do Olho Seco em Ginecologia. Moreira Jr.

Williams, D., et al. (2012). Comparison of hyaluronic acid-containing topical eye drops with carbomer-based topical ocular gel as a tear replacement in canine keratoconjunctivitis sicca: A prospective study in twenty five dogs. Veterinary Research Forum, 3(4): 229 - 232. 